\title{
Multidimensional Poverty Analysis: Looking for a Middle Ground
}

Francisco H. G. Ferreira • Maria Ana Lugo

Widespread agreement that poverty is a multifaceted phenomenon encompassing deprivations in multiple dimensions clashes with the vociferous disagreement about how best to measure these deprivations. Drawing on the recent literature, this short paper reviews three methodological alternatives to the false dichotomy between scalar indices of multidimensional poverty, on the one hand, and a "dashboard" approach that considers only marginal distributions, on the other. These alternatives include simple Venn diagrams of the overlap of deprivations across dimensions, multivariate stochastic dominance analysis, and the analysis of copula functions, which capture the extent of interdependency across dimensions. Examples are provided from the literature on both developing and developed countries. JEL codes: I32, O15

Over the last 10 years, interest in multidimensional poverty measurement has grown steadily. Since the pioneering works of Bourguignon and Chakravarty (2003) and Tsui (2002), a number of approaches have been proposed to measure or analyze deprivation in multiple dimensions. This rapidly growing literature now includes Alkire and Foster (2011a), Chakravarty, Deutsch, and Silber (2008), Deutsch and Silber (2005), Duclos, Sahn, and Younger (2006), and Maasoumi and Lugo (2008), among others.

Multidimensional poverty analysis has been transformed from a purely academic discussion into a broad domestic and international policy debate, both within and between many countries. In December 2009, for example, Mexico's National Council for the Evaluation of Social Policy (CONEVAL) adopted a multidimensional index as the country's official poverty measure. ${ }^{1}$ In 2011, the government of Colombia followed suit by adopting a poverty-reduction strategy that focused on five separate dimensions and relied on a variant of Alkire and Foster's (2011a) 
approach to quantify progress in the reduction of poverty. Internationally, the Multidimensional Poverty Index of Alkire and Santos (2010), which was reported for over 100 countries in the UNDP's Human Development Report 2010, has also gained prominence (UNDP 2010).

The driving force behind this rising popularity is the acknowledgment that poverty involves much more than just low income. Low consumption and inadequate living standards are at the heart of the concept of "poverty," to be sure. The associations conjured up by that term also include aspects of poor health, such as a shortened lifespan; limited access to education, knowledge, and information; and powerlessness in various domains. Poor people often mention nonincome dimensions as being crucial to their perceptions of their own hardships. The following quote from a person from Georgia illustrates this point: "Poverty is lack of freedom, enslaved by crushing daily burden, by depression and fear of what the future will bring" (quoted in Narayan et al. 2000, p. 37). It is now quite common for poverty analysts, whether from academia, the World Bank, or other agencies, to face enquiries about how best to summarize information on "multidimensional poverty" in a particular country.

However, the various new multidimensional poverty indices have not been universally welcomed. Serious criticism of these indices centers on the manner in which information on deprivation is aggregated across dimensions. One powerful critique is that the weights used to aggregate across dimensions lack the intrinsic meaning associated with prices, which are used to add the components of consumption expenditure (or, implicitly, its dual, the incomes used to finance consumption). Under the law of one price, and given relatively weak assumptions on preferences, relative prices are equal to the rate at which consumers themselvesregardless of their income levels and allowing for different utility functions-are willing to trade one such component (e.g., bread) for another (e.g., a bicycle).

Of course, there are a number of practical reasons why prices may not be ideal welfare weights. These reasons range from the existence of externalities to the fact that price data are often geographically coarse, so actual price variation in space is missing from the information available to the researcher. Nevertheless, as Ravallion (2011, p. 247) argues,

It is widely agreed that prices can be missing for some goods and deceptive for others. There are continuing challenges facing applied economists in addressing these problems. However, it is one thing to recognize that markets or prices are missing or imperfect, and quite another to ignore them in welfare and poverty measurement. There is a peculiar inconsistency in the literature on multidimensional indices of poverty whereby prices are regarded as an unreliable guide to the tradeoffs, and are largely 
ignored, while the actual weights being assumed in lieu of prices are not made explicit in the same space as prices.

Because multidimensional indices adopt arbitrary weights, which are often equal weights across dimensions, they inherently involve specific tradeoffs between the constituent components of welfare in the mathematical sense: A certain extra amount of one component will exactly offset the change in another component to leave the index unchanged. These tradeoffs are seldom stated explicitly, and it is not obvious that they are frequently revised by public debate (Ravallion 2011).

\section{What Is the Disagreement?}

The debate between proponents and skeptics of multidimensional poverty measurement was featured in the Forum Section of the June 2011 issue of the Journal of Economic Inequality (e.g., Alkire and Foster 2011b; Ravallion 2011; Lustig 2011). These articles suggest strong agreement on at least one basic point: "poverty is multidimensional" (Ravallion 2011, p. 236). There is little dispute that deprivation exists in multiple domains, which are often correlated. Therefore, considering information on these various dimensions (rather than on incomes or consumption expenditures only) is likely to be useful for designing policies that effectively combat poverty.

The disagreement between proponents and skeptics involves how best to measure this multidimensional poverty - that is, how best to convey information about the extent of these various deprivations in a way that is useful for both analysts and policy makers. Some studies, such as those by Alkire and Foster (2011a) and Maasoumi and Lugo (2008), have proposed scalar indices that seek to combine information from various dimensions into a single number. A key advantage of such scalar indices is that they generate a complete ordering of countries, regions, or individuals, even when the rankings conflict across individual dimensions. The Multidimensional Poverty Index (or the Human Development Index, in the space of attainments) attracts a good deal of international attention, in large part because it ranks countries according to how well they perform on various dimensions through a simple summarizing tool.

In contrast, Ravallion (2011) suggests a "dashboard" approach, whereby "we may need to focus our efforts and resources on developing the best possible distinct measures of the various dimensions of poverty [...] aiming for a credible set of 'multiple indices' rather than a single 'multidimensional index'" (Ravallion 2011, p. 13; our emphasis). An important limitation of multidimensional indices, as noted earlier, is that they require the use of relative weights for each dimension, which are chosen somewhat arbitrarily by the analyst. Other analysts, policy makers, and the public may disagree with these specific weights. Instead of 
imposing a specific weighting system, the dashboard approach allows the user to attach more or less importance to any particular dimension.

\section{Is There a Policy-relevant Middle Ground for Multidimensional Poverty Analysis?}

In this short paper, we argue that this debate-a single index versus a dashboard-is a false dichotomy. In other words, we suggest that the analysis of multidimensional poverty can and should move beyond both scalar indices and dashboards of deprivation based on marginal distributions. Multidimensional poverty analysis is interesting because the joint distribution of achievements provides more information than that provided by its margins. The dependency structure in a joint distribution-how closely correlated various achievements (or deprivations) are - can affect how we assess poverty in a society or how we compare it across time periods, even given identical margins. A dashboard that reports on poverty indices for each dimension separately may overlook this aspect of the joint distribution.

This point was made eloquently by Duclos, Sahn, and Younger (2006). In one instance, these authors compare the joint distributions of two dimensions of health (nutrition and survival probabilities) in Cameroon and Madagascar and find that both marginal distributions in Cameroon first-order dominate the corresponding marginal distributions in Madagascar. However, there is no dominance of the joint distribution. In this example, a dashboard approach would lead a researcher to naively conclude that poverty was unambiguously greater in Madagascar, whereas a truly multidimensional assessment (i.e., one that took into account the correlations) would enable the researcher to conclude that no clear ranking was possible.

The discrepancy arises because the correlation between the two dimensions may differ substantially from one place to another, so the cumulative concentration of deprivations could make overall poverty worse in a place that has better marginal distributions. As Duclos et al. put it, "It is possible for a set of univariate analyses done independently for each dimension of well-being to conclude that poverty in A is lower than poverty in B while a multivariate analysis concludes the opposite, and vice-versa. The key to these possibilities is the interaction of the various dimensions of well-being in the poverty measure and their correlation in the sampled populations" (Duclos, Sahn and Younger 2006, p. 945).

In the remainder of this paper, we briefly describe three alternative empirical approaches for the analysis of the dependency structure in joint distributions. All three illustrations are drawn from the recent literature, and we make no claim to 
originality. Each is suitable to a specific purpose, but all of the approaches focus on interactions among the dimensions, so the menu of options may be useful not only to analysts but also to policy makers.

The first approach is the set of multivariate stochastic dominance techniques proposed by Duclos et al. (2006), which enable poverty analysts to investigate joint distributions of multiple deprivations without making the specific assumptions about tradeoffs that understandably worry Ravallion (2011). If the correlation between deprivations matters - as surely it must - then this approach must be regarded as superior to the dashboard approach, which considers only the marginal distributions.

Multivariate stochastic dominance compares two multidimensional distributions to determine whether one distribution dominates the other (i.e., consistently lies always above or always below) for all reasonable poverty frontiers. If this is the case, then one could conclude that poverty in, say, A is always higher (or lower) than poverty in B for all additive poverty indices. Below, we reproduce two examples given in Duclos et al. (2006). These graphs represent the bidimensional dominance surfaces-that is, the difference between two joint distributions.

The graph on the left of Figure 1 represents the difference in the surfaces of two hypothetical distributions. In this case, "[a]though differences in the univariate dominance curves in both dimensions clearly cross the origin (at the extreme left and right of the figure), there is a significant interior section where the first surface is entirely above the second" (Duclos et al. 2006, p. 954). In other words, there is intersection dominance without marginal dominance.

Figure 1. Differences in Dominance Surfaces
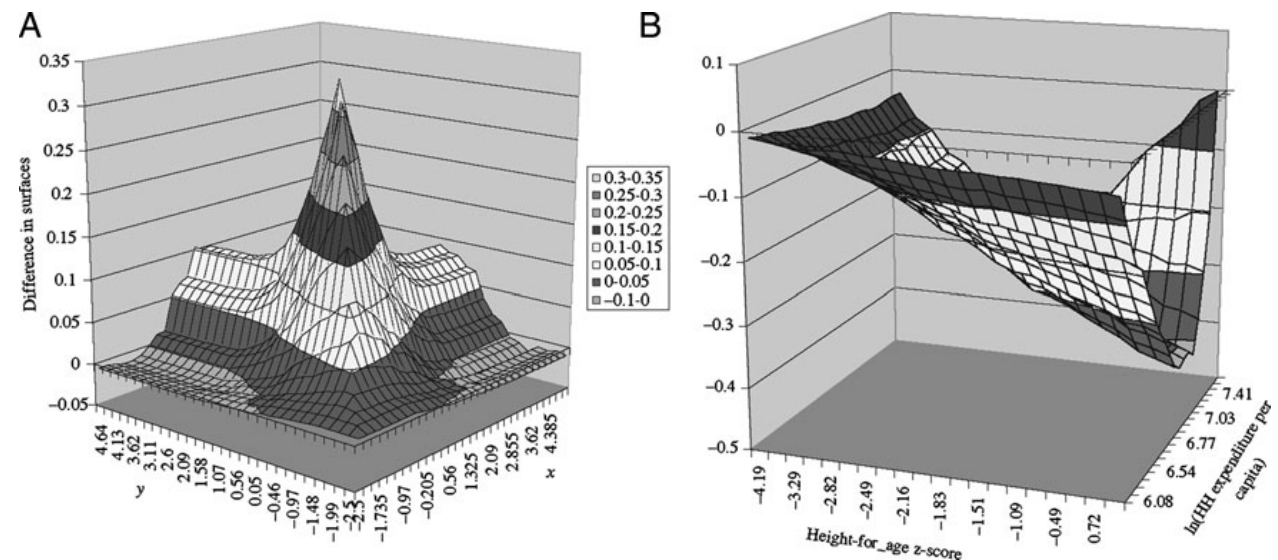

Source: Reprinted from Duclos, J.-Y., D. Sahn, and S. Younger. 2006. "Robust Multidimensional Poverty Comparisons.” Economic Journal 116 (514): 943-68. 
The graph on the right of Figure 1 depicts the dominance surface comparing rural and urban children in Vietnam on two dimensions: household expenditure per capita and height-for-age $\mathrm{Z}$ scores. This case indicates that over almost the entire range of expenditures and stunting, rural children are poorer than their urban counterparts. This dominance is found for all reasonable poverty lines, so the finding that "rural children are poorer than urban ones is valid for almost any intersection, union or intermediate poverty frontier" (Duclos et al. 2006, p. 959).

Of course, it is true that multivariate stochastic dominance analysis tends to be of limited use when the number of dimensions or margins is large. In these cases, a seldom-discussed natural alternative involves complementing the dashboard approach with a direct representation of the dependency structure. In poverty studies, the degree of interdependence can be presented in terms of the extent of the overlap between individuals who are identified as deprived under the various criteria. For instance, if poverty were defined by three dimensions (such as education, health, and income), the dependency could be illustrated - at least in partby the proportion of individuals who were deprived in all three dimensions, those who were deprived in (different) pairs of dimensions, or those who were deprived in only one dimension. Atkinson and Lugo (2010) provide such an example for the case of Tanzania.

Table 1 below (reproducing Table 5 in Atkinson and Lugo 2010) presents information on deprivations in three dimensions of well-being measured at the household level and the extent of overlap between them. The dimensions chosen are school attendance of children between 5 and 16 years old, access to safe sources of drinking water (piped or protected), and an indicator of ownership of durable assets. These indicators are closely related to the goals set by the government in the Tanzanian National Strategy and are obtained at the household level from the (same) Household Budget Surveys. The example shows the following:

Between 2001 and 2007, school attendance and availability of durable assets have improved significantly, whereas access to protected sources of drinking water has deteriorated. Despite the latter, the combined effect is to reduce the proportion of Tanzanians who suffer from any of the three forms of deprivation: this has fallen from 90 per cent to 80 per cent. Equally there has been a fall in the proportion deprived on all 3 dimensions: from 19 per cent to 10 per cent. At the same time, there has been an increase in one category of the deprived: those lacking only access to water (Atkinson and Lugo 2010, p. 15).

The implication of these results for assessing the progress of the country between the two years is clear: "If it were decided that access to water were the sole concern, then deprivation would have increased from 46 per cent in 2001 to 51 
Table 1. Deprivations in schooling, access to protected water and durable assets in Tanzania

\begin{tabular}{lrr}
\hline Proportion of the individuals living in households ... & 2001 & 2007 \\
\hline School deprived: at least one child 5-16 years old not in school & 55.2 & 34.5 \\
Assets deprived: no car and fewer than on "small asset" & 66.6 & 47.4 \\
Water deprived: no access to piped or protected source of drinking water & 45.8 & 50.7 \\
Distributions of individuals & & \\
Not deprived in school, water or assets & 10.3 & 20.1 \\
Only school deprived & 8.9 & 7.5 \\
Only water deprived & 4.7 & 14.4 \\
Only assets deprived & 17.3 & 15.1 \\
School and water deprived & 9.5 & 10.7 \\
Water and assets deprived & 12.5 & 15.9 \\
School and assets deprived & 17.6 & 6.6 \\
School, water and assets deprived & 19.1 & 9.8 \\
\hline
\end{tabular}

Source: HBS 2001 and 2007. Reproduced from Atkinson and Lugo (2010), "Growth, poverty and distribution in Tanzania." International Growth Centre, Working Paper 10/0831. November. Page 15.

Note: small assets include television, radio, telephone (including mobile phones), refrigerator bicycle, and motorcycle.

per cent in 2007. [...] [If instead, one wanted to] assess overall performance simply in terms of the proportion deprived on all dimensions [...] this proportion has fallen, indicating definite progress" (Atkinson and Lugo 2010, p. 15).

An effective way of diagrammatically presenting this kind of information on the degree of overlap across dimension-specific deprivations is through Venn diagrams, as Atkinson et al. (2010) do for the EU-27, the 27 countries of the European Union. The larger the overlap between deprivations is, the greater the extent of interdependence will be. Figure 2 (reprinted from Atkinson et al. 2010) shows the number of people who are at risk of poverty (EU definition), the number of people who are materially deprived, and the number of people aged 0-59 years who are living in jobless households. ${ }^{2}$ Data come from European Union Statistics on Income and Living Conditions surveys. The authors note, "A little over 80 million people live in households at risk of poverty, a further 40 million live in households that are not at risk of poverty but are defined as jobless and/or materially deprived." Indeed, "well over two-thirds are identified under only one of the criteria." This especially insightful example suggests that, in this particular context, policies that are directed exclusively toward one of the indicators may fail to reduce the degree of deprivation of a large proportion of households. The authors astutely conclude that it is important not only to monitor the three indicators but also to understand that the degree of overlap among them will help to shape policies to address these shortfalls. 
Figure 2. Multiple Indicators from the Europe 2020 Target

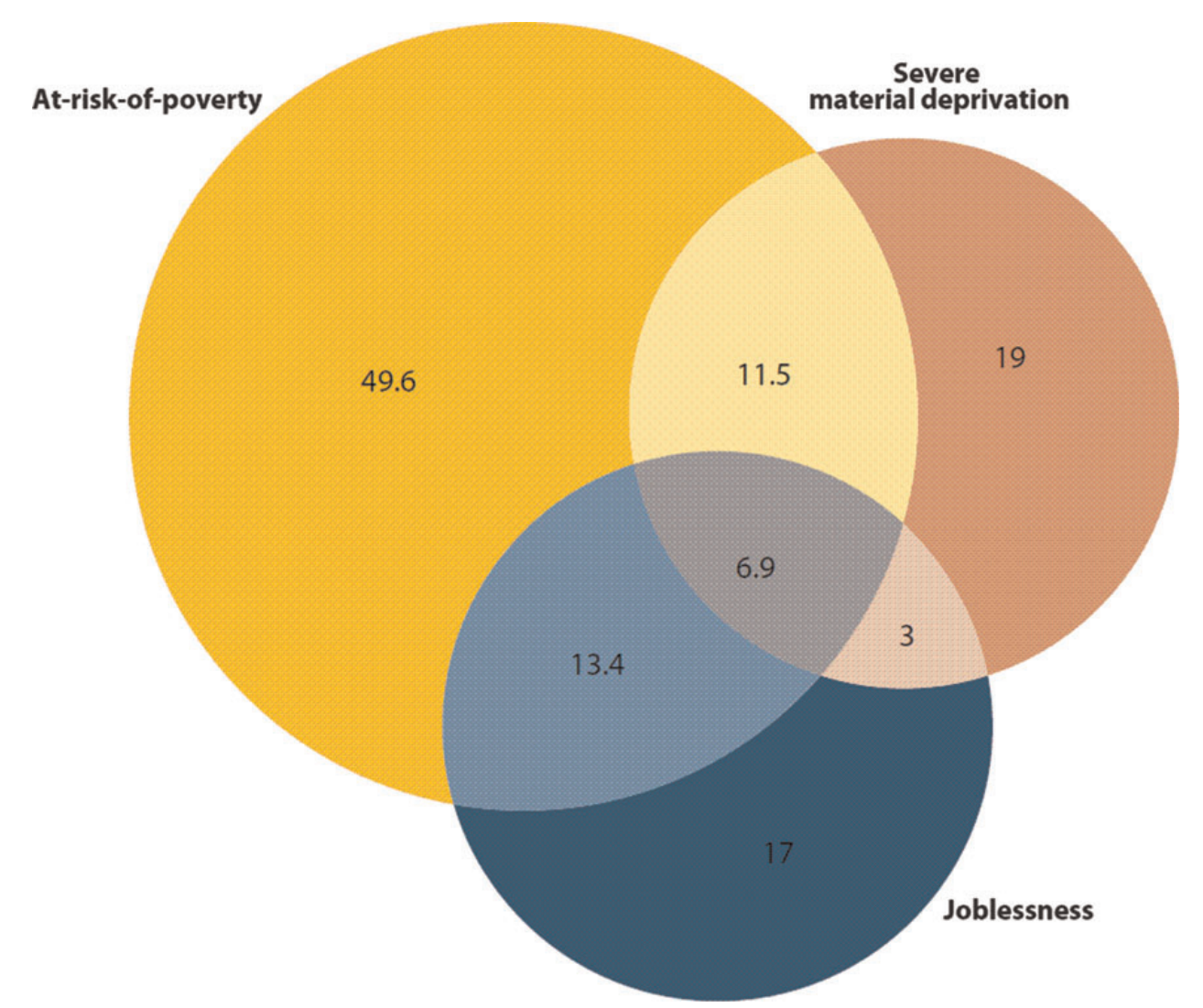

Note: Figures for EU-27 in million of persons.

Source: EU-SILC, Eurostat-CEPS/INSTEAD calculations. Reprinted from Atkinson, A. B., E. Marlier, F. Monatigne, and A. Reinstadler. 2010. "Income Poverty and Income Inequality." In A.B. Atkinson and E. Marlier, eds., Income and Living Conditions in Europe, 127. Luxemburg: Eurostat.

When the objective is to evaluate well-being rather than deprivation, the representation of interdependencies between dimensions is less straightforward, and some "lateral thinking" (Atkinson 2011) might be necessary. Sklar's Theorem, from the statistics literature, tells us that any joint distribution function can be decomposed into the marginal distribution functions of each dimension (corresponding to the dashboard approach) and a copula function, which captures the degree of interdependency between the dimensions. Copulas, which are the third approach we want to highlight, have been used to study the relationship between health and income (Decancq 2009, Quinn 2007). Formally, the copula function is the joint distribution function of $n$ vectors whose elements are the relative position of every individual in each of the $n$ dimensions of well-being. As in the case 
Figure 3. The Evolution of the Dependence between the Dimensions of Well-being over Time in Russia

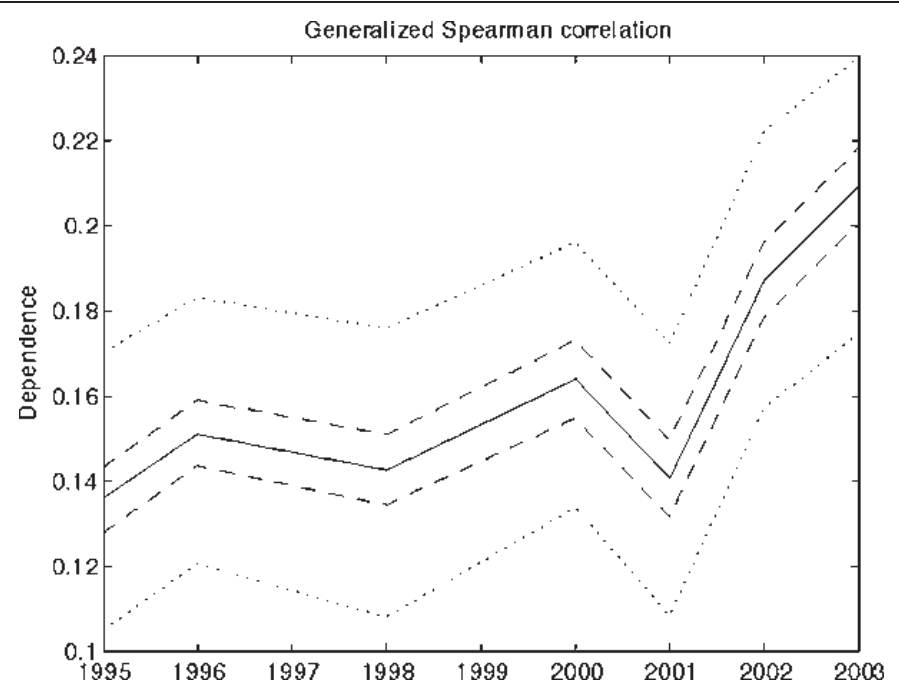

Source: RLMS 1995-2003. Reprinted from Decancq, K. 2009. "Copula-based Measurement of Dependence Between Dimensions of Well-being." HEDG Working Paper 09/32. University of York, Health Economics Resource Centre, York, UK.

of the margins, once the copula function is constructed, a stochastic dominance analysis could be performed to assess whether the degree of interdependency between the components has changed in an unambiguous way. In addition, and in the same way that information on unidimensional inequality can be summarized by an index (such as the Gini coefficient or the mean log deviation), measures of rank correlation allow us to order distributions unambiguously in terms of their degree of interdependency (Decancq 2009).

As an example, we reproduce from Decancq (2009) a graph showing the evolution of the Spearman correlation coefficient, a commonly used measure of rank correlation, in Russia between 1995 and 2003 (Figure 3). The author considers three dimensions, standard of living, health, and schooling, represented respectively by household income, self-assessed health, and years of schooling as primary ranking variables (three other variables are used as secondary ranking variables in case of ties). For context, the Russian Human Development Index increased from the beginning to the end of the period, albeit with a deterioration around the time of the 1998 financial crises.

In this figure, the dashed line represents the 95 percent confidence interval obtained by Monte Carlo simulations, whereas the dotted line represents an alternative computation of the same confidence interval by bootstrapping. The figure 
shows that the degree of dependence across these three dimensions of well-being has increased throughout most of the period; as average well-being was rising, so was the degree of interdependency between the dimensions of well-being.

Although they differ in technical complexity, these three alternative techniques (Venn diagrammatic representations of the dependency structure, multivariate stochastic dominance, and the analysis of copula functions) seem to avoid the disadvantages associated with both the scalar indices and the dashboard approach. Like the dashboard approach (but unlike scalar indices), these techniques do not require the use of generally arbitrary weights to aggregate across dimensions, with their unpalatable implications in terms of tradeoffs. Like scalar indices (but unlike the dashboard approach), these techniques incorporate information about how deprivations are jointly distributed and allow analysts to take into account different levels or changes in the extent of overlap or correlation between them. This is a menu of analytical approaches that represents an advantageous middle ground in multidimensional poverty analysis and that may add value to some of the poverty analyses currently undertaken at the World Bank (and elsewhere).

There is one important caveat, however. This kind of analysis requires that information on the various dimensions be observed for each unit of observation (typically, the individual or the household). In other words, the dimensions must be observed in the same survey (or census) or, at least, in different surveys that cover the same set of households and contain common identifiers. Otherwise, it is clearly impossible to observe the joint distribution.

An especially important argument proposed by Ravallion (2011) for the dashboard approach is that the best data on separate dimensions (say, health status and consumption expenditures) are often found in different data sets, from which no joint distribution can be constructed. In these cases, a tradeoff may arise between data quality and information on the joint distribution, and such tradeoffs must be evaluated on a case-by-case basis. If the quality of information is thought to differ only marginally - if, for example, anthropometric information about children in a health survey is thought to be superior to that contained in an Living Standards Measurement Study-type survey, but the latter has some informationsome analysts may be sufficiently interested in the extent of the overlap of deprivation in health and consumption to incur the cost of using the slightly worse data. Conversely, analysts who place sufficient weight on the accuracy of the information on marginal distribution would choose to analyze each margin separately, drawing from the best survey in each case. ${ }^{3}$

In many cases, of course, reliable information on relevant dimensions, such as health status, anthropometrics, education, and consumption, exists in the same survey. This is the case, for instance, for the Russia Longitudinal Monitoring Survey used by Decancq (2009), the Indonesia Family Life Survey, and various Living Standards Measurement Study-type surveys. In these cases, it is difficult to 
find a justifiable excuse for any analyst who fails to complement his marginal analysis with information on the dependency structure. Furthermore, one should not underestimate the power of the demand for better data. If there are certain aspects of well-being and deprivation that are not regularly captured in household surveys but whose joint distribution with other dimensions (such as income) is of real policy interest, then more frequent analysis of the kind we suggest might encourage statistical institutes or other data providers to collect information on such aspects.

\section{What about Scalar Multidimensional Poverty Indices?}

Finally, we turn briefly to the question of whether the analytical approaches we propose should completely preclude the computation of scalar multidimensional poverty indices. It is clear that they do not preclude the dashboard approach in that considering the marginal distributions is inherent in considering the joint distributions. The three approaches described above are best understood as complementing the dashboard approach with information on the dependency structure between the dimensions, when this information is available.

What role do we see for the definition and computation of scalar multidimensional poverty indices? We see this question as another instance of choice given a tradeoff. A scalar multidimensional index provides a complete ordering, with the ability to rank two years, countries, or regions, even when their joint distributions (or copulas) cross. Just as in unidimensional poverty or inequality analysis, the ability to generate a complete ordering comes at a cost in terms of specific functional form assumptions. In the case of multidimensional well-being and poverty, this price is high, for two key reasons.

The first reason is that the identification step (in the sense of Sen 1976) is considerably more complex for multidimensional poverty than for unidimensional poverty. This complexity results not only because one has to define a threshold for each individual dimension but also because a difficult choice must be made at the identification step about how many deprivations constitute poverty. This situation recalls the fundamental choice between the union and intersection approaches (e.g., Bourguignon and Chakravarty 2003), the establishment of an intermediate cutoff in the number of dimensions (Alkire and Foster 2011a), or whether the depth of deprivation in one dimension should be allowed to offset well-being in another dimension at the identification stage (e.g., Duclos et al. 2006). In this paper, we have largely ignored issues of identification, although these issues are clearly important if one decides to pursue the scalar-index route.

The second reason is the need for weights to aggregate across dimensions. Even conditional on a particular identification algorithm, the issue of weights remains. 
Whether a price is worth paying is therefore likely to depend on, first, the importance of the ability to rank for the purpose of the analysis at hand and, second, the arbitrariness (and number) of the weights.

Therefore, it is difficult to take a definitive position in the abstract. As in the case of unidimensional poverty measurement, it seems that two extreme positions are untenable. The first position would be to say that a particular multidimensional poverty index is the one true measure of poverty. The second position would be to argue that any particular index that makes an unpalatable assumption is inadmissible. In the same way that we recognize the limitations of the headcount index but continue to report it (ideally, alongside other measures and more disaggregated and robust analysis), there are clear uses for various indices of multidimensional poverty.

In our view, such scalar indices would be most useful if they relied, to the greatest possible extent, on (shadow or market) prices to aggregate across different goods and services. Only the aspects of well-being for which there can truly be no sensible estimate of relative prices should be treated as separate dimensions. Although food, cooking utensils, toilets, clothing, and vehicles may be resources that affect different "functionings" (in the sense of Amartya Sen), they are best treated as components of a single dimension of well-being-command over private goods - whose internal weights are given by relative prices.

Equilibrium prices, as noted earlier (and in elementary first-year economics courses), reflect people's preferences and constraints as well as market structures. If individual choices can be reasonably approximated by the result of maximizing a utility function subject to a budget constraint, and if the law of one price holds, then it follows that (regardless of people's individual utility functions or income levels) the rate at which every person trades off one unit of a good against one unit of another good will be the same. In other words, relative prices reflect the marginal rate of substitution between the goods.

Naturally, price data are often problematic. Very often, the available data on prices do not accurately represent the prices actually encountered by individuals for the indicators that we would include in the analysis. This is largely because there is price heterogeneity across regions (or even cities) within a country and in the quality of products and services, whereas price data tend to be collected at a higher level of aggregation and for a representative (average) item in each category. However, it is questionable whether these problems are so severe that choosing arbitrary weights across dimensions, such as the availability of cooking utensils and, say, the ownership of certain means of transport, would yield a preferable metric. In this regard, Ravallion's (2011) arguments for greater reliance on market prices seem overwhelmingly compelling.

The true value of the analysis of multidimensional poverty (or well-being) lies in the existence of certain aspects of well-being that we deem important but for which there can be no sensible estimate of relative prices. It is reasonable to 
include in this category items such as political and personal freedoms, health, and, arguably, education. Although various inputs into the production of health and education are marketable, this is not true of all of them. Health is influenced by environmental quality and by a number of other public goods. Education, once embodied in human capital, generates so many externalities that it is difficult to think that school fees or costs are suitable approximations of its true shadow price. These things - not the material from which a ceiling is made or the kind of stove one uses-are the true dimensions of welfare.

In these cases, multidimensional analysis becomes particularly relevant. If, for the purpose at hand, the analyst decides that the price of selecting weights is worth paying (to obtain a complete ordering), then the question is whether one can choose relative weights that at least attempt to represent the existing tradeoff between the different components of deprivation (or well-being). There are various approaches to setting these weights; some are based exclusively on the observed distribution of attributes, others are based on people's opinions, and others use both sorts of information (Decancq and Lugo, 2013). Regardless of the weighting scheme and the precise functional form chosen, multidimensional indices of deprivation (or well-being) should be allowed to be sensitive to the essence of the multidimensional approach, that is, to the degree of dependency between its components.

\section{Conclusions}

There is widespread agreement that poverty is a multifaceted phenomenon. Income shortfalls, which translate into an inability to consume certain basic commodities, are central to this phenomenon. However, income poverty is typically associated with deprivation in other realms, such as health, education, social status, and political power, which are more difficult to price. These associations or correlations between the constituent dimensions of poverty vary over time and from place to place, and they are often believed to be significant. Recent advances in multidimensional poverty analysis seek to capture these interactions, and revealed preference seems to suggest that they are of interest to policy makers in many developing countries.

Despite this widespread agreement on the essential fact that poverty is multidimensional, there has been a lively debate about whether this implies that scalar indices should be constructed that summarize information on these various dimensions into a single number or whether multiple indices should be provided, one for each dimension, in a dashboard approach. Drawing on the existing literature, we have argued here that such a dichotomous view misses the point. The most interesting aspects of the multidimensionality of poverty arise from the 
interdependence among dimensions. The joint distribution of dimensions over the population contains more information than the corresponding marginal distributions, and the correlation patterns in that joint distribution may change how we compare poverty across two countries or time periods. This dependency structure is overlooked entirely by the dashboard approach and is often obscured by scalar indices.

Drawing on examples from both developed and developing countries, we provide three alternative approaches that allow researchers and policy analysts to focus on the dependency structure of a joint distribution. The first approach is stochastic dominance analysis, which permits partial orderings across joint distributions that are robust not only to poverty lines and welfare weights (as in the unidimensional case) but also to dimension weights. The second approach is a representation of the overlap of deprivations over the population by means of simple tabulations or Venn diagrams. Given the agreement on the identification criterion along each dimension, this extremely simple tool can complement the dashboard statistics on the marginal distributions in informative ways. The third approach involves the use of copula functions to study the multivariate association among different components of well-being across two or more joint distributions.

Finally, we argue that multidimensional poverty indices, like most other tools, can be accommodated in the economist's toolkit, and the risk of serious injury decreases with reliance on relative prices and a focus on a few core, truly irreducible dimensions. If such indices are used, fodder will remain for future controversies on the choice of weights, poverty lines, and functional forms, but these controversies would largely be a distraction from what really matters to policy makers: the pattern of associations and overlaps across the core dimensions of well-being.

\section{Notes}

Francisco H. G. Ferreira is with the Development Research Group at the World Bank and the Institute for the Study of Labour (IZA); email address: fferreira@worldbank.org. Maria Ana Lugo is in the Poverty, Equity and Gender Group at the Latin America Region of the World Bank. This paper is an expanded version of a short comment that was published as Ferreira (2011). We are grateful to three anonymous referees and to Sabina Alkire, Peter Lanjouw, Nora Lustig, and Martin Ravallion for comments on earlier versions of the paper. We are also grateful for illuminating conversations with James Foster on this subject. All remaining errors are ours. This paper is a product of the Equity and Development Research Project (P099861). The views expressed here are those of the authors. They should not be attributed to the World Bank, its Executive Directors, or the countries they represent.

1. The Executive Secretary of the National Council for the Evaluation of Social Policy, Dr. Gonzalo Hernández Licona, is quoted as saying, "Mexico is proud to be the first country in the world to measure poverty, not narrowly on economic grounds alone, but to take full account of crucial social components of poverty such as quality of housing and access to healthcare and food, 
which are all too often neglected by established poverty measures." See http://www.ophi.org.uk/ launch-of-mexico $\%$ E2\%80\%99s-new-poverty-measure/.

2. Persons "at risk of poverty" are defined as those who have an equivalized disposable income below 60 percent of the national median equivalized disposable income, after social transfers. Material deprivation covers indicators relating either to economic strain or to the ownership of durables. Severely materially deprived persons cannot afford at least four of the following: to pay rent or utility bills; to keep their home adequately warm; to pay unexpected expenses; to eat meat, fish, or a protein equivalent every second day; a one-week holiday away from home; a car; a washing machine; a color TV; or a telephone. Finally, a "jobless household" is one in which none of the members aged 18-59 years are working or the members aged 18-59 years have very limited work attachment.

3. The question of whether data on a particular variable are more reliable in one survey than in another may, in some cases, be investigated empirically. At a minimum, survey-to-survey imputation techniques can be used to assess the sensitivity of results to using information from different sources. This remains an area for future work.

\section{References}

Alkire, S., and J. Foster. 2011a. "Counting and Multidimensional Poverty Measurement." Journal of Public Economics 95 (7): 476-87.

. 2011b. "Understandings and Misunderstandings of Multidimensional Poverty Measurement.” Journal of Economic Inequality 9 (2): 289-314.

Alkire, S., and M.E. Santos. 2010. "Acute Multidimensional Poverty: A New Index for Developing Countries.” OPHI Working Paper Series \#38. Oxford University, Department of International Development, Oxford Poverty and Human Development Initiative, Oxford, UK.

Atkinson, A.B. 2011. "On Lateral Thinking.” Journal of Economic Inequality 9 (3): 319-28.

Atkinson, A.B., E. Marlier, F. Monatigne, and A. Reinstadler. 2010. "Income Poverty and Income Inequality." In A. Atkinson and E. Marlier, eds., Income and Living Conditions in Europe 287-398. Luxemburg: Eurostat.

Atkinson, A.B., and M.A. Lugo. 2010. "Growth, Poverty and Distribution in Tanzania." International Growth Centre Working Paper 10/0831. London School of Economics and Political Science and Oxford University, Department for International Development, London and Oxford, UK.

Bourguignon, F., and S. Chakravarty. 2003. "The Measurement of Multidimensional Poverty." Journal of Economic Inequality 1 (1): 25-49.

Chakravarty, S., J. Deutsch, and J. Silber. 2008. "On the Watts Multidimensional Poverty Index and its Decomposition.” World Development 36 (6): 1067-77.

Decancq, K. 2009. “Copula-Based Measurement of Dependence Between Dimensions of Well-Being.” HEDG Working Paper 09/32. University of York, Health Economics Resource Centre, York, UK.

Decancq, K., and M.A. Lugo. 2013. "Weights in Multidimensional Indices of Well-Being: An Overview." Econometric Reviews 32 (1): 7-34.

Deutsch, J., and J. Silber. 2005. "Measuring Multidimensional Poverty: An Empirical Comparison of Various Approaches." Review of Income and Wealth 51 (1): 145-74.

Duclos, J.-Y., D. Sahn, and S. Younger. 2006. "Robust Multidimensional Poverty Comparisons." Economic Journal 116 (514): 943-68.

Ferreira, F.H.G. 2011. "Poverty is Multidimensional. But What Are We Going To Do About It?" Journal of Economic Inequality 9 (3): 493-95. 
Lustig, N. 2011. "Multidimensional Indices of Achievements and Poverty: What Do We Gain and What Do We Lose? An Introduction to the JOEI Forum on Multidimensional Poverty." Journal of Economic Inequality 9 (2): 227-34.

Maasoumi, E., and M.A. Lugo. 2008. "The Information Basis of Multivariate Poverty Assessments." In N. Kakwani and J. Silber, eds., Quantitative Approaches to Multidimensional Poverty Measurement. New York: Palgrave Macmillan.

Narayan, D., R. Patel, K. Schafft, A. Rademacher, and S. Koch-Schulte. 2000. Voices of the Poor: Can Anyone Hear Us? Washington, DC: World Bank.

Quinn, C. 2007. "Using Copulas to Measure Association between Ordinal Measures of Health and Income." HEDG Working Paper 07/24. University of York, Health Economics Resource Centre, York, UK.

Ravallion, M. 2011. "On Multidimensional Indices of Poverty." Journal of Economic Inequality 9 (2): $235-48$.

Sen, A.K. 1976. "Poverty: An Ordinal Approach to Measurement." Econometrica 44 (2):219-31.

Tsui, K.-Y. 2002. “Multidimensional Poverty Indices.” Social Choice and Welfare 19 (1): 69-93.

UNDP. 2010. Human Development Report 2010: The Real Wealth of Nations: Pathways to Human Development. New York: Palgrave Macmillan. 